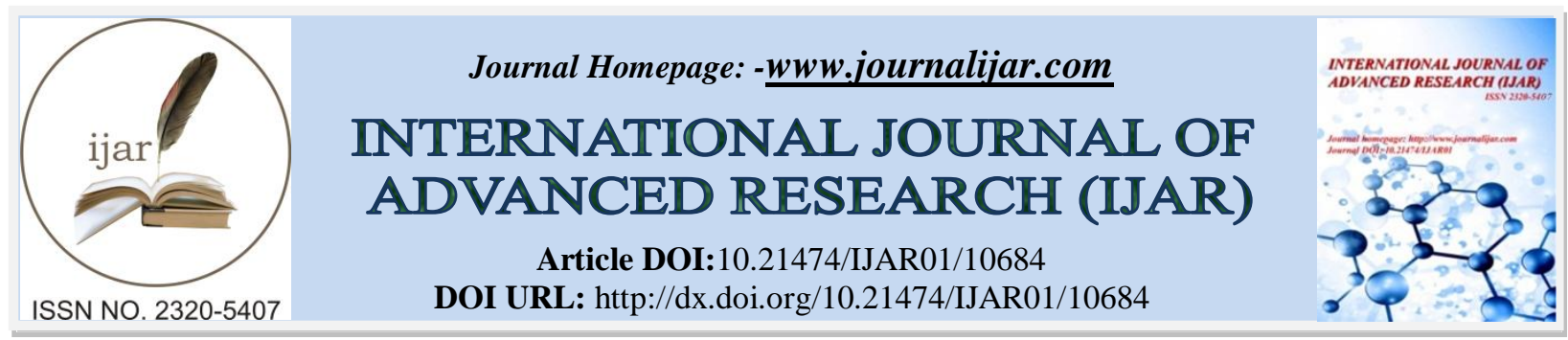

RESEARCH ARTICLE

\title{
ASSESSMENT OF EFFECT OF STORAGE TIME ON PLATELET CONCENTRATION OF PRF: IN
} VITRO STUDY

\section{Dr. Anuja Hudwekar}

\section{Manuscript Info}

\section{Manuscript History}

Received: 17 January 2020

Final Accepted: 20 February 2020

Published: March 2020

Key words:-

Platelet Rich Fibrin, Blood Platelets, Storage Time

\section{Abstract}

Introduction:The ability of PRF to sustain its platelet concentrate over the tested time may have significant clinical implications. Blood drawn before the start of the procedure and stored as PRF improves patient comfort and saves operator time, since no specialised equipment or storage facilities are required.

Aim: To determine whether storage time has a significant effect on the platelet concentration of platelet-rich fibrin (PRF).

Materials and Methods: A total of 10 healthy participants were enrolled into the study. From all the participants, three separate blood samples were collected by venepuncture. Two of the blood samples were centrifuged to form PRF. Formed PRF was removed from the respective test tubes at different time intervals - immediately after centrifugation (Group A) and after $60 \mathrm{~min}$ of storage time in the blood collecting tube (Group B). The residual blood from each group was tested for platelet concentration and comparison was made with noncentrifuged baseline sample (Group C).

Results: The residual mean platelet concentration of Group A was higher than that determined for Group B. The results from this study indicate that there was a significant difference in the mean platelet concentration of PRF when stored for 0 and 60 minutes.

Conclusion : The study concluded that clot disintegration does not take place after 15 minutes of storage, but rather remains stable over a period of at least 60 minutes and may in fact increase in platelet concentration during that time.

Copy Right, IJAR, 2020,. All rights reserved.

\section{Introduction:-}

The development of bioactive surgical additives, which are being used to regulate the inflammation and increase the speed of healing process $^{(1)}$, is one of the great challenges in clinical research. In this sense, healing is a complex process, which involves cellular organization, chemical signals, and the extracellular matrix for tissue repair ${ }^{(2)}$. The understanding of healing process is still incomplete, but it is well known that platelets play an important role in both haemostasis and wound healing processes ${ }^{(3)}$.

Platelets' regenerative potential was introduced in the 70 's ${ }^{(4)}$, when it was observed that they contain growth factors that are responsible for increase collagen production, cell mitosis, blood vessels growth, recruitment of other cells that migrate to the site of injury, and cell differentiation induction, among others ${ }^{(5) .}$ 
The most common platelet concentrate used in these procedures is platelet-rich plasma (PRP) and by the early part of the 21 st century, its use in various surgical procedures was commonplace. Whitman et al ${ }^{(6)}$, in 1997 , were the first to introduce the use of platelet-rich plasma in oral surgical procedures,However, using it also presents risk because bovine thrombin, which is used to handle PRP, may generate antibodies to factors $\mathrm{V}, \mathrm{XI}$, and thrombin that could cause coagulopathies that may endanger life ${ }^{5}$. On the other hand, PRF was first used in 2001 by Choukroun et al. $^{\text {(7) }}$, specifically in oral and maxillofacial surgery, and is currently considered as a new generation of platelet concentrate. It consists of a matrix of autologous fibrin and has several advantages over $\mathrm{PRP}^{(1)}$, including easier preparation and not requiring chemical manipulation of the blood, which makes it strictly an autologous preparation ${ }^{(5)}$. It is now regarded as a biological scaffold different to PRP. However, even though the use of PRF is gaining widespread clinical acceptance, several questions regarding its biological stability remain unanswered. The aim of this this study was to determine whether storage time has a significant effect on the platelet concentration of platelet-rich fibrin (PRF).

\section{Materials and Methods:-}

The present study is cohort analytical study carried in department of Periodontics, M.G.Vs KBH Dental College, Nashik. The study was explained to the patients, informed consent was obtained. The study protocol was approved by the Institutional Ethical Committee of M.G.Vs KBH Dental College, Nashik.

A total of 10 healthy participants were enrolled into the study. From all the participants, three separate blood samples were collected by venepuncture. Two of the samples were acquired using tubes containing clot activators, i.e., VacuetteR 10ml serum tubes with Z Serum Clot Activator (Greiner BioOne International AG, Germany), whereas the remaining blood sample was drawn into a $10 \mathrm{ml}$ BD VacutainerR tube that contained dipotassiumEDTA, an anticoagulant (BD Diagnostics, New Jersey USA).

All the blood samples collected in the anticoagulant containing tubes were used to measure baseline platelet concentrations fig. 2, whereas the remaining blood samples (collected in the Clot Activator containing tubes) were used to prepare PRF by centrifugation (400g for 12 minutes) in a standard benchtop centrifuge (PLC-03, Hicare International, Taiwan) (fig 1). Therefore, from each study participant, two samples of PRF were obtained. The two PRF samples were then randomly allocated into either Group A or Group B (fig.3 \& 4), using a simple coin toss.

For Group A, the PRF was removed from the tube immediately after preparation (0min), whereas for Group B, the PRF remained in the tube and was only removed after 60 minutes. The residual blood from each group was tested for platelet concentration and comparison was made with non-centrifuged baseline sample (Group C).

We calculated the platelet concentration of PRF for each group indirectly, by determining the numerical difference between the residual platelet concentration (of the remaining serum after removal of PRF) and the baseline platelet concentration for each specific study participant.In this study, platelet concentration analysis was carried out using an electronic automated cell counter.

Data was collected and entered into a spreadsheet (Microsoft Office 2010 Excel, Microsoft Corporation, Washington). The results were compared and analysed statistically using SPSSR Version 13 for Windows.

\section{Results:-}

A total of 10 participants ( 5 females and 5 males) were entered into the study. The mean age of the participants was 40 years. The youngest participant was 20 years old and the oldest, 54 years old. Platelet concentrations obtained from analysis of all 10 participants were within the normal laboratory reference range of $170-400 \times 10^{9} \mathrm{~L}$ of circulating blood. The mean blood platelet concentration was $280.4 \times 10^{9} / \mathrm{L}$ (Table 1).There was no significant difference between the genders.

After removal of formed PRF from Group A, the residual serum yielded minimal concentrations of platelets. The mean concentration of remaining platelets was $7.5 \times 10^{9} / \mathrm{L}$. (Table 2)

For Group B, serum platelet concentrations were also minimal after removal of the prepared PRF with the mean concentration of the platelets being $4.0 \times 10^{9} /$ L. (Table 2 ) 
The residual mean platelet concentration of Group A was higher than that determined for Group B with the mean difference in platelet concentrates between the two groups being $3.50 \times 109 / \mathrm{L}$. Using a non-parametric Signed Rank Test, the statistical significance of the differences in platelet concentrates between the Groups A and B was analysed. The difference between the two groups was statistically significant $(\mathrm{p}<0.001)$. The platelet concentration of PRF for both groups was calculated using the difference between baseline and residual platelet concentrations. (Table 3) A paired t-test showed a statistically significant difference between the two groups $(\mathrm{p}<0.001)$ (Table 4)

\section{Discussion:-}

The aim of this this study was to determine whether storage time has a significant effect on the platelet concentration of platelet-rich fibrin (PRF).Structural properties of PRF does not allow for direct measurement of its platelet concentration.

We calculated the PRF concentrationfor each group indirectly, by determining the numerical difference between the residual platelet concentration (of the remaining serum after removal of PRF) and the baseline platelet concentration for each specific study participant.Higher residual values indicate that less platelets were present in the removed clot whereas lower residual values would indicate that more platelets were present in the clot. When differences between the test groups and the baseline blood sample were compared, it was clear

that $97 \%-98 \%$ of the platelets were concentrated in the PRF clot. The results of present study are in accordance with study done by DohanEhrenfest et al (2010). PRF has a natural fibrin framework with growth factors within that may keep their activity for a relatively longer period and stimulate tissue regeneration effectively ${ }^{(8,9)}$.

Previous research has indicated that storing PRF under certain conditions may affect its ability to clinically yield positive results. In fact it is not recommended to store platelet concentrate in its blood collecting tube, as the serum has not been flushed away from the clot, the growth factor content remains stable. It is a good way to gain 5-15 minutes, but this is not a long-term solution, because the clot slowly starts to sink into the tube after centrifugation and to merge with the red blood cell base, leading to an unusable material loaded with red blood cells and with weak mechanical properties. Instead several authors propose to keep the clots in a sterile metal cup and to press them into membranes with a sterile metal spoon when needed. Data regarding the maximal storage time and the ideal storage temperature of PRF are however largely lacking ${ }^{(8)}$.

The present study used standard blood collecting tubes with clot activators to store PRF for at least 60 minutes at room temperature. As the $60 \mathrm{~min}$. of duration time was chosen on the basis of average time of any periodontal surgical procedure at the faculty of dentistry. The results of present study showed that it was possible to concentrate more than $97 \%$ of available blood platelet into a readily usable form and also storing the concentrate for 60 minutes resulted in a form of PRF that had significantly higher concentrations of platelets compared with non-stored PRF.

The reason for this phenomenon may be related to clot activators. Due to the lack of an anticoagulant, blood begins to coagulate as soon as it comes in contact with the glass surface. The contact with a silica surface is required to activate the clot polymerization process ${ }^{(10)}$. Finally, platelets seem to be trapped massively in the fibrin meshes. Therefore, for successful preparation of PRF, speedy blood collection and immediate centrifugation before the initiation clotting cascade is absolutely essential. Quick handling is the key to achieve a clinically usable PRF clot ${ }^{(11)}$. Another reason for the variation in platelet concentration may be that the recommended centrifuge time is too short to allow for complete clot formation to occur. Therefore, allowing the PRF clot to remain in the tube for a period longer than the recommended time, may result in a more complete physiological reaction taking place.

The ability of PRF to sustain its platelet concentrate over the tested time may have significant clinical implications. Instead of drawing the blood during surgical procedure, it allows for blood to be drawn before the start of procedure this improves the patients comfort and save the operators time. To store the PRF in same tube avoid its exposure to the environmental factors. It is also cost-effective, since no specialised equipment or storage facilities are required. This may be necessary in resource-poor settings.

The release of growth factors is a significant property of blood platelets. Some studies showed that PRP had limited effect to stimulate bone regeneration ${ }^{(12,13)}$. It is hypothesized that PRP releases growth factors quickly, just before the cell outgrowth from the surrounding tissu $\mathrm{e}^{(14)}$. Platelet-rich fibrin is prepared naturally without addition of thrombin, and it is hypothesized that PRF has a natural fibrin framework and can protect growth factors from proteolysis.Thus, growth factors can keep their activity for a relatively longer period and stimulate bone 
regeneration effectively. The platelet counts of PRF and PRP were similar, it is shown that PRF can release growth factors gradually and keep their activity to a relatively long period compared with PRP ${ }^{(15)}$.

In the present study, it was shown that the platelet concentration of PRF improved over a period of 60 minutes of storage. We assume that this may be due to prolonged fibrin clot maturation. It is therefore reasonable to speculate that if PRF is stored until optimal fibrin formation is achieved, then higher concentrations of growth factors may be available from the PRF during wound healing. Whether this has any clinical significance requires further investigation.

\section{Limitation:}

1. Limited number of sample size.

2. The inability to directly measure the platelet concentration of PRF

3. The small difference between the platelet concentrations of the groups tested. Although statistically significant, a mean difference.

\section{Future perspect:}

1. Therefore further research is warranted to determine the clinical significance of these findings.

\section{Conclusion:-}

This study showed that it is possible to concentrate more than $97 \%$ of the available platelets into a PRF clot. The study also indicated that, unlike in previous reports, clot disintegration does not take place after 15 minutes of storage, but rather remains stable over a period of at least 60 minutes and may in fact increase in platelet concentration during that time.

\section{References:-}

1. Dohan DM, Choukroun J, Diss A, Dohan SL, Dohan AJ, Mouhyi J and Gogly B. Platelet-rich fibrin (PRF): a second-generation platelet concentrate. Part I: technological concepts and evolution. Oral Surg Oral Med Oral Pathol Oral RadiolEndod 2006; 101: e37-44.

2. Singer AJ and Clark RA. Cutaneous wound healing. N Engl J Med 1999; 341: 738-746.

3. Gassling VL, Açil Y, Springer IN, Hubert N and Wiltfang J. Platelet-rich plasma and platelet-rich fibrin in human cell culture. Oral Surg Oral Med Oral Pathol Oral RadiolEndod 2009; 108: 48-55.

4. Ross R, Glomset J, Kariya B and Harker L. A platelet-dependent serum factor that stimulates the proliferation of arterial smooth muscle cells in vitro. ProcNatlAcadSci U S A 1974; 71: 1207-1210.

5. Kiran NK, Mukunda KS and Tilak Raj TN. Platelet concentrates: A promising innovation in dentistry. J Dent Sci Res 2011; 2: 50-61.

6. Whitmann DH, Berry RL and Green DM. Platelet gel: an alternative to fibrin glue with applications in oral and maxillofacial surgery. J Oral MaxillofacSurg 1997; 55: 1294-1299.

7. Choukroun J, Adda F, Schoeffler C and Vervelle A. Uneopportunité en paro-implantologie: le PRF. Implantodontie 2000; 42: 55-62.

8. DohanEhrenfest, D M, Del Corso, M, Diss, A, et al. Three-dimensional architecture and cell composition of a Choukroun's platelet-rich fibrin clot and membrane. Journal of Periodontology.2010; 81 (4); 546-55.

9. Dohan,DM, Choukroun,J, Diss,A, et al. Platelet-rich fibrin (PRF): a second-generation platelet concentrate. Part II:platelet-related biologic features. Oral Surgery, Oral Medicine,Oral Pathology, Oral Radiology, and Endodontology.2006; 101 (3); 45-50.

10. Dohan DM, Del Corso M and Charrier J-B. Cytotoxicity analyses of Choukroun's platelet-rich fibrin (PRF) on a wide range of human cells: The answer to a commercial controversy. Oral Surg Oral Med Oral Pathol Oral RadiolEndod 2007; 103: 587-58.

11. Anilkumar K, Geetha A, Umasudhakar, Ramakrishnan T, Vijayalakshmi R, Pameela E. Platelet-rich-fibrin: A novel root coverage approach. J Indian SocPeriodontol 2009;13: 50-4.

12. Schlegel KA, Donath K, Rupprecht S, Falk S, Zimmermann R,Felszeghy E, Wiltfang J. De novo bone formation using bovinecollagen and platelet-rich plasma. Biomaterials 2004;25:5387-93.

13. Thorwarth M, Rupprecht S, Falk S, Felszeghy E, Wiltfang J,Schlegel KA. Expression of bone matrix proteins during de novobone formation using a bovine collagen and platelet-rich plasma(PRP) - an immunohistochemical analysis. Biomaterials 2005;26:2575-84. 
14. Lacoste E, Martineau L, Gagnon G. Platelet concentrates: effect of calcium and thrombin on endothelial cell proliferation andgrowth factor release. J Periodontol 2003;74:1498-507.

15. Lundquist R, Dziegiel MH, Agren MS. Bioactivity and stability of endogenous fibrogenic factors in plateletrich fibrin. WoundRepair Regen 2008;16:356-63.

\section{Result Tables:}

Table 1. Mean baseline platelet concentration in cell $\times 10^{9} / \mathrm{L}$ of blood

\begin{tabular}{|l|l|l|}
\hline & mean & SD \\
\hline Male & 2.75 & 40 \\
\hline Female & 2.85 & 65 \\
\hline
\end{tabular}

Table 2. Mean baseline platelet concentration of residual blood in cell $\times 10^{9} / \mathrm{L}$ of blood

\begin{tabular}{|l|l|l|l|l|}
\hline & Mean & $\mathrm{N}$ & $\mathrm{SD}$ & Std. error mean \\
\hline Group A & 7.5 & 10 & 3.01 & 0.55 \\
\hline Group B & 4 & 10 & 1.90 & 0.33 \\
\hline
\end{tabular}

Table 3. Mean calculated PRF platelet concentration in cell $\times 10^{9} / \mathrm{L}$ of blood

\begin{tabular}{|l|l|l|}
\hline & Mean & SD \\
\hline PRF A & 2.72 & 57 \\
\hline PRF B & 2.76 & 58 \\
\hline
\end{tabular}

Table 4. Statistical analysis of PRF A and PRF B (Paired $t$ test, $P$ value is significant)

\begin{tabular}{|l|l|l|l|l|l|l|l|}
\hline Mean & SD & $\begin{array}{l}\text { STD } \\
\text { error mean }\end{array}$ & \multicolumn{2}{l|}{$\begin{array}{l}\text { 95\% } \\
\text { confidence interval }\end{array}$} & $\mathrm{t}$ & df & Significance \\
\hline & & & $\mathrm{L}$ & $\mathrm{U}$ & & & \\
\hline-3.9 & 3.13 & 0.57 & -5.0 & -2.7 & -6.18 & 29 & 0.001 \\
\hline
\end{tabular}


Figure 1. PRF Preparation

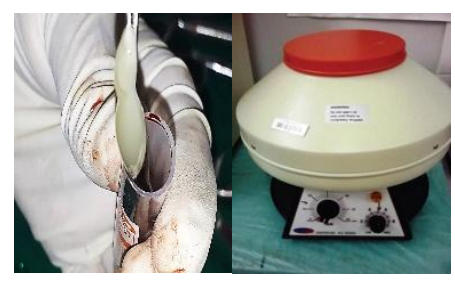

Figure 3. Group A platelet concentration immediate after PRFafter storage of PRF

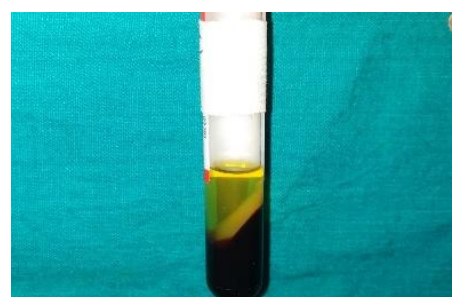

Figure 2. Baseline platelet concentration

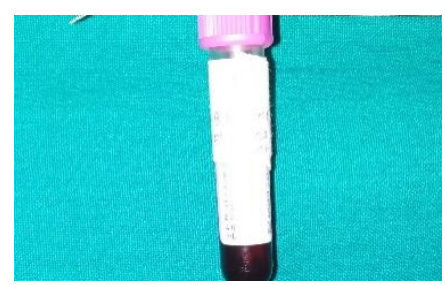

Fig.4 Group B platelet concentration 60 min.

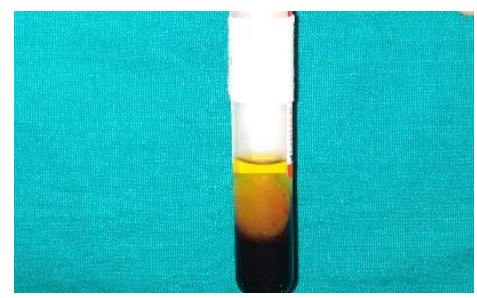

